\title{
Simple and computationally efficient movement classification approach for EMG- controlled prosthetic hand: ANFIS vs. artificial neural network
}

\begin{abstract}
The aim of this paper is to propose an exploratory study on simple, accurate and computationally efficient movement classification technique for prosthetic hand application. The surface myoelectric signals were acquired from 2 musclesð Flexor Carpi Ulnaris and Extensor Carpi Radialis of 4 normal-limb subjects. These signals were segmented and the features extracted using a new combined time-domain method of feature extraction. The fuzzy C-mean clustering method and scatter plots were used to evaluate the performance of the proposed multi-feature versus other accurate multi-features. Finally, the movements were classified using the adaptive neuro-fuzzy inference system (ANFIS) and the artificial neural network. Comparison results indicate that ANFIS not only displays higher classification accuracy $(88.90 \%)$ than the artificial neural network, but it also improves computation time significantly.
\end{abstract}

Keyword: Pattern recognition; EMG; ANFIS; Neural network; Prosthetic hand 